\title{
Line Ratio Diagnostics Applicable to Astronomical Spectra in the 50-3000 $\AA$ Wavelength Region
}

\author{
F. P. KEENAN \\ Department of Pure and Applied Physics, The Queen's University of Belfast, \\ Belfast BT7 $1 \mathrm{NN}, \mathrm{N}$. Ireland
}

\begin{abstract}
A bibliography has been produced of the most reliable emission and absorption line ratio diagnostic calculations currently available for application to the spectra of astrophysical sources in the UV and EUV wavelength region (50-3000 $\AA$ ). References are listed containing diagnostics for species in the Li through $\mathrm{P}$ isoelectronic sequences, as well as the iron ions Fe II-Fe XXIII and nickel ions Ni XVII-Ni XXV. Also given is the wavelength range for which diagnostic calculations are presented in each reference, along with the type of diagnostic considered. These include, for example, emission line ratios for determining electron temperatures and densities, and absorption line diagnostics for evaluating hydrogen densities.
\end{abstract}

\section{Introduction}

Line ratios involving transitions in the ultraviolet (UV) and extreme ultraviolet (EUV) regions of the spectrum frequently provide excellent temperature and density diagnostics for the emitting or absorbing plasma. Over the past 20 years, many such diagnostics have been developed for application to astronomical spectra, such as those of the solar transition region/corona (Dere 1978; Vernazza \& Reeves 1978), and stellar and interstellar observations from the International Ultraviolet Explorer and Copernicus satellites (Hyung, Aller \& Feibelman 1994; Jenkins \& Shaya 1979; Jordan 1988). More recently, new observing opportunities, such as those afforded by the Hubble Space Telescope (HST), Extreme Ultraviolet Explorer (EUVE), Orbiting and Retrievable Far Ultraviolet Telescope (ORFEUS), and Hopkins Ultraviolet Telescope (HUT), have lead to a large increase in both the quality and quantity of astronomical UV and EUV observations, and there is clearly an urgent requirement for diagnostics which may be applied to the analysis of such data.

There have been several recent reviews on the importance of diagnostics for analysing UV and EUV astronomical spectra (Dwivedi 1994; Feldman \& Doschek 1991; Feldman et al. 1992). However of particular importance is that of Mason \& Monsignori Fossi (1994), which discusses the spectroscopic techniques used to study astrophysical plasmas, the atomic processes involved, recent observations and plans for future space missions. My aim therefore is to complement the Mason \& Monsignori Fossi review by providing a bibliography of the most reliable emission and absorption line ratio diagnostics currently available for transitions observable in the UV and EUV spectra of astronomical sources. Such diagnostics will not only be applicable to observations from, for example, Skylab, HST, EUVE, ORFEUS, and HUT, but will also be useful for analysing data from upcoming missions such as the Solar and Heliospheric Observatory (SOHO).

\section{Bibliography of Diagnostic Calculations}

I have compiled a list of references to the most reliable diagnostic calculations currently available for application to astronomical spectra in the UV and EUV wavelength 
range, 50-3000 $\AA$. Species are listed by isoelectronic sequence, apart from ions of Fe and $\mathrm{Ni}$ which are considered separately. After each species, I summarise the wavelength range for which diagnostic calculations are presented in the relevant reference. In some instances, references contain diagnostics involving transitions over a wider wavelength range than that considered here; in these instances $I$ list the full wavelength coverage of the diagnostics. For example, the Fe XIII diagnostic calculations of Young, Mason \& Thomas (1995) consider not only the EUV lines at $\sim 202 \AA$, but also the IR transitions at $\sim 1.08 \mu \mathrm{m}$, while Cai \& Pradhan (1993) similarly provide data for both UV ( $\sim 910 \AA)$ and optical $(\sim 6731 \AA$ ) lines in S II. Also given is a list which indicates the type of diagnostic calculated in the relevant reference. The vast majority of the diagnostics are emission line electron temperature or density diagnostics; this is not surprising as most work in this area has been performed in order to analyse solar or nebular emission line spectra.

Omitted from the lists are diagnostics for H-like, He-like and F-like ions, as to the best of my knowledge there are no calculations for these sequences applicable to spectra in the 50-3000 $\AA$ wavelength range, apart from electron temperature sensitive emission line ratios involving the He II 256-304 $\AA$ lines (Cassinelli et al. 1995) and the Ar X 165-4257 $\AA$ transitions (Feldman \& Doschek 1977). Nor do I list diagnostics that involve lines from different elements, as these are rather limited in number (see, for example, Keenan, Dufton \& Kingston 1990).

I would like to apologize in advance to anyone whose work has been inadvertently omitted from the tables. For the future, I would be grateful if readers would inform me of such omissions, and also of any of their more recent diagnostic work that could be included in future versions of these tables.

Full copies of the bibliography, which includes 15 tables and 156 references, are available from the author either by post or by electronic mail (from F.Keenan@qub.ac.uk on Internet).

I would like to thank all those who sent me reprints/preprints of their work for inclusion in this paper, including L. Aller, A. Bhatia, J. Cassinelli, G. Doschek, B. Dwivedi, U. Feldman, S. Kastner, H. Mason, J. Peng, A. Pradhan and H. Zhang.

\section{REFERENCES}

Car, W. \& Pradhan, A. K. 1993, ApJS, 88, 329

CAssinelli, J. P. et al., 1995 ApJ, 438, 932

Dere, K. P. 1978, ApJ, 221, 1062

Dwivedi, B. N. 1994, Space Sci. Rev., 65, 289

Feldman, U. \& DoscheK, G. A. 1977, J. Opt. Soc. Am., 67, 726

Feldman, U. \& DoscheK, G. A. 1991, ApJS, 75, 925

Feldman, U., Mandlebaum, P., Seely, J. F., Doschek, G. A. \& GuRsky, H. 1992, ApJS, 81,387

Hyung, S., Aller, L. H. \& Feibelman, W. A. 1994, PASP, 106, 745

Jenkins, E. B. \& Shaya, E. J. 1979, ApJ, 231, 55

JordAN, C. 1988, J. Opt. Soc. Am. B, 5, 2252

Keenan, F. P., Dufton, P. L. \& Kingston, A. E. 1990, ApJ, 353, 636

Mason, H. E. \& Monsignori, Fossi, B. C. 1994, A\&AR, 6, 123

Vernazza, J. E. \& Reeves, E. M. 1978, ApJS, 37, 485

Young, P. R., Mason, H. E. \& Thomas, R. J. 1995, Proc. 3rd SOHO Workshop, in press 